\title{
Extreme Nausea due to Hyperparathyroidism in Multiple Endocrine Neoplasia-1
}

${ }^{1}$ TK Pandian, ${ }^{2}$ Geoffrey Thompson, ${ }^{3}$ DY Benzon

\section{ABSTRACT}

We describe a young woman with multiple endocrine neoplasia-1 (MEN-1) who presented with severe nausea as a result of hyperparathyroidism (HPT). We present a case report, review the relevant literature in regards to physiology, and offer a target source for symptom control.

A 19-year female with MEN-1 initially presented with neuroglycopenia and hyperinsulinism. She had concomitant HPT. She underwent a distal pancreatectomy with enucleation of neuroendocrine tumors from the pancreatic head. Postoperatively, she developed severe nausea and was found to have worsening HPT. She was placed on a calcimimetic and had immediate resolution of symptoms. She ultimately underwent a subtotal parathyroidectomy and has been symptom-free since that time.

Severe nausea can occur in up to $25 \%$ of patients with HPT. Two theories exist regarding the mechanism behind this symptomatology. The first involves destruction of gastric mucosa leading to peptic ulcer disease. The second suggests that the etiology is gastrointestinal atony due to high calcium levels or parathyroid hormone (PTH). Based on the available data, therapies to treat nausea in HPT should focus on decreasing high levels of calcium.

Keywords: Hypercalcemia, Hyperparathyroidism, Multiple endocrine neoplasia-1, Nausea, Parathyroidectomy.

How to cite this article: Pandian TK, Thompson G, Benzon DY. Extreme Nausea due to Hyperparathyroidism in Multiple Endocrine Neoplasia-1. World J Endoc Surg 2015;7(3):69-71.

Source of support: Nil

Conflict of interest: None

\section{INTRODUCTION}

Gastrointestinal manifestations of hyperparathyroidism (HPT) have been noted since the earliest description of the disease in the early 1900s. ${ }^{1,2}$ The most commonly associated symptoms include constipation, heartburn, nausea and loss of appetite. Nausea, specifically, has been described in up to $25 \%$ of patients with HPT. ${ }^{2,3}$ Nausea and resultant emeses can be quite debilitating, and may even mimic hyperemesis gravidum in pregnancy. ${ }^{4,5}$ The physiologic mechanism of nausea in HPT is not fully understood.

\footnotetext{
${ }^{1}$ Surgical Trainee, ${ }^{2}$ Professor, ${ }^{3}$ Associate Professor

${ }^{1-3}$ Department of Subspecialty General Surgery, Mayo Clinic Minnesota, United States
}

Corresponding Author: Geoffrey Thompson, Professor Department of Subspecialty General Surgery, Mayo Clinic Minnesota, United States, e-mail: thompson.geoffrey@mayo.edu

\section{CASE REPORT}

A 19-year old woman who was part of a multiple endocrine neoplasia type 1 (MEN-1) kindred family and with known menin protein mutation presented to our clinic is a 19-year-old woman with a known menin protein mutation and is part of a multiple endocrine neoplasia type 1 (MEN-1) kindred family. She developed neuroglycopenic symptoms and was evaluated with a 72-hour fast. The results were positive for hyperinsulinism. Subsequent imaging revealed multiple masses in the pancreatic head and tail consistent with neuroendocrine tumors (examples in Figs 1A and B). Her diagnostic work-up also included a pituitary magnetic resonance imaging (MRI) which was negative, as well as a serum
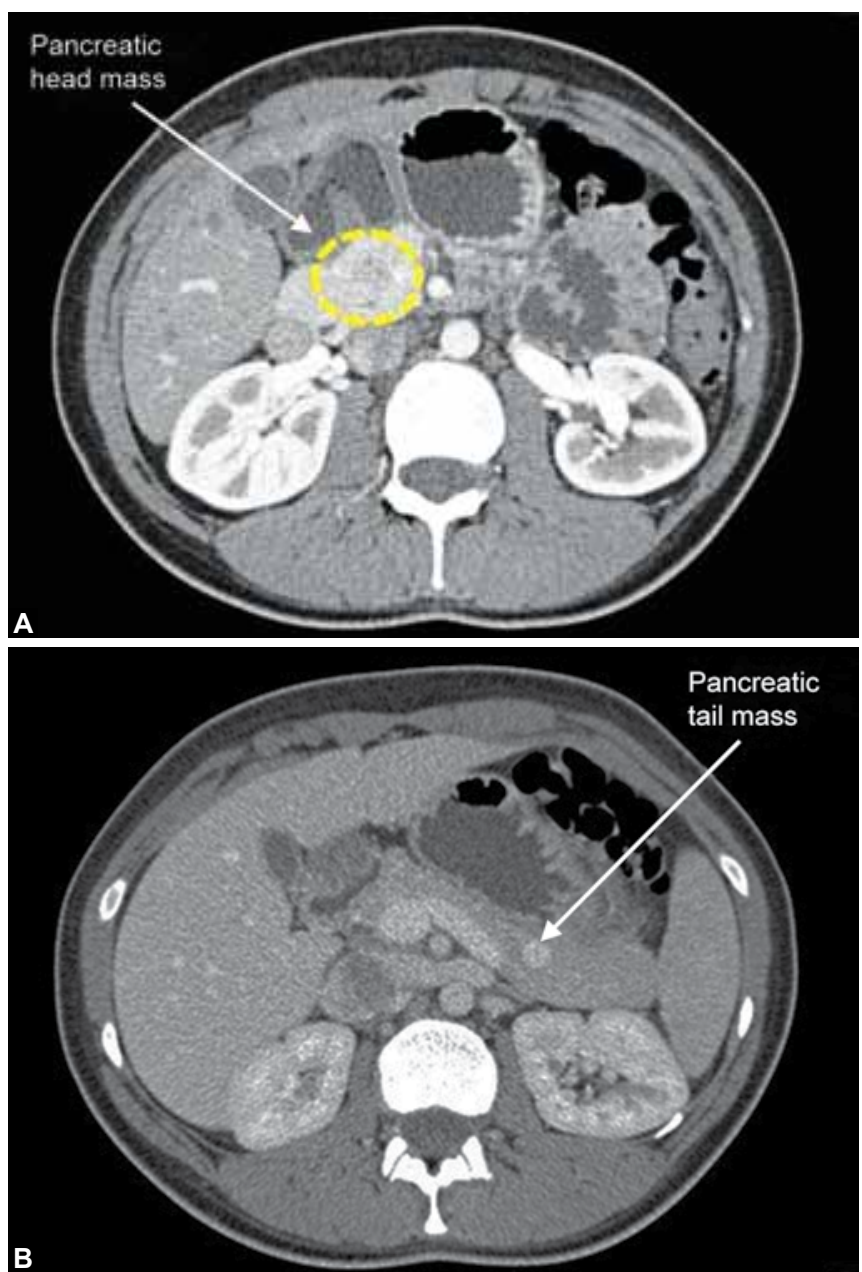

Figs 1A and B: Axial CT abdomen: (A) Pancreatic head mass-an ill-defined, hypodense pancreatic head lesion is denoted by the dashed yellow line, (B) pancreatic tail mass-a contrast-enhanced pancreatic tail lesion is noted by the arrow 
calcium of $10.8 \mathrm{mg} / \mathrm{dl}$ (normal: $8.9-10.1 \mathrm{mg} / \mathrm{dl}$ ) and parathyroid hormone (PTH) level of $68 \mathrm{pg} / \mathrm{ml}$ (normal: $15-65 \mathrm{pg} / \mathrm{ml}$ ) consistent with primary HPT without overt clinical symptoms. Due to her neuroglycopenia, she underwent an Ann Arbor Procedure with extended distal pancreatectomy and enucleation of lesions within the pancreatic head.

Postoperatively, she developed severe and persistent nausea. At 4 and 6 weeks, computed tomography (CT) scans of her abdomen and pelvis showed no abnormalities or fluid collections. Six weeks postoperatively, her serum calcium level was $11.6 \mathrm{mg} / \mathrm{dl}$ and PTH was $78 \mathrm{pg} / \mathrm{ml}$. A trial of cinacalcet was given and her nausea immediately resolved. A sestamibi localization scan revealed parathyroid hyperplasia (Fig. 2). She underwent subtotal parathyroidectomy (Fig. 3) shortly thereafter and has been asymptomatic at 3 months follow-up.

\section{DISCUSSION}

The physiologic mechanisms behind gastrointestinal symptomatology of HPT have not been fully elucidated. Whether the primary culprit is PTH itself or the resultant hypercalcemia has yet to be definitively proven. Presence of symptoms with concurrent elevations of both molecules makes delineation difficult. In the late 1960s, there was some speculation that sustained PTH levels may affect gene expression resulting in altered gastric mucus and ensuing destruction of gastric mucosa. ${ }^{6}$ This was shown in some animal models in vivo, however was not reproducible in humans. ${ }^{6}$ The ultimate downstream effect of mucosal damage is peptic ulcer disease (PUD), which has been associated with hyperparathyroid states in historical studies. ${ }^{6-9}$ Although the major symptom of PUD is abdominal pain, nausea can occur as well.

Hypercalcemia (both within and outside the setting of HPT) has been associated with a number of gastrointestinal manifestations including anorexia, nausea, abdominal pain, and constipation..$^{10}$ Nausea and hypercalcemia have been linked to gastrointestinal atony. ${ }^{1,7-9}$ The specific mechanism involves two concurrent processes. First, increased levels of calcium impede transmission of afferent stimuli and diminish efferent discharges due to constant amounts of acetylcholine in the sympathetic ganglia of the gut. In addition, a high calcium ion concentration at the neuromuscular junction of these ganglia alters the membrane potential and leads to reduced neuromuscular excitability. Collectively,

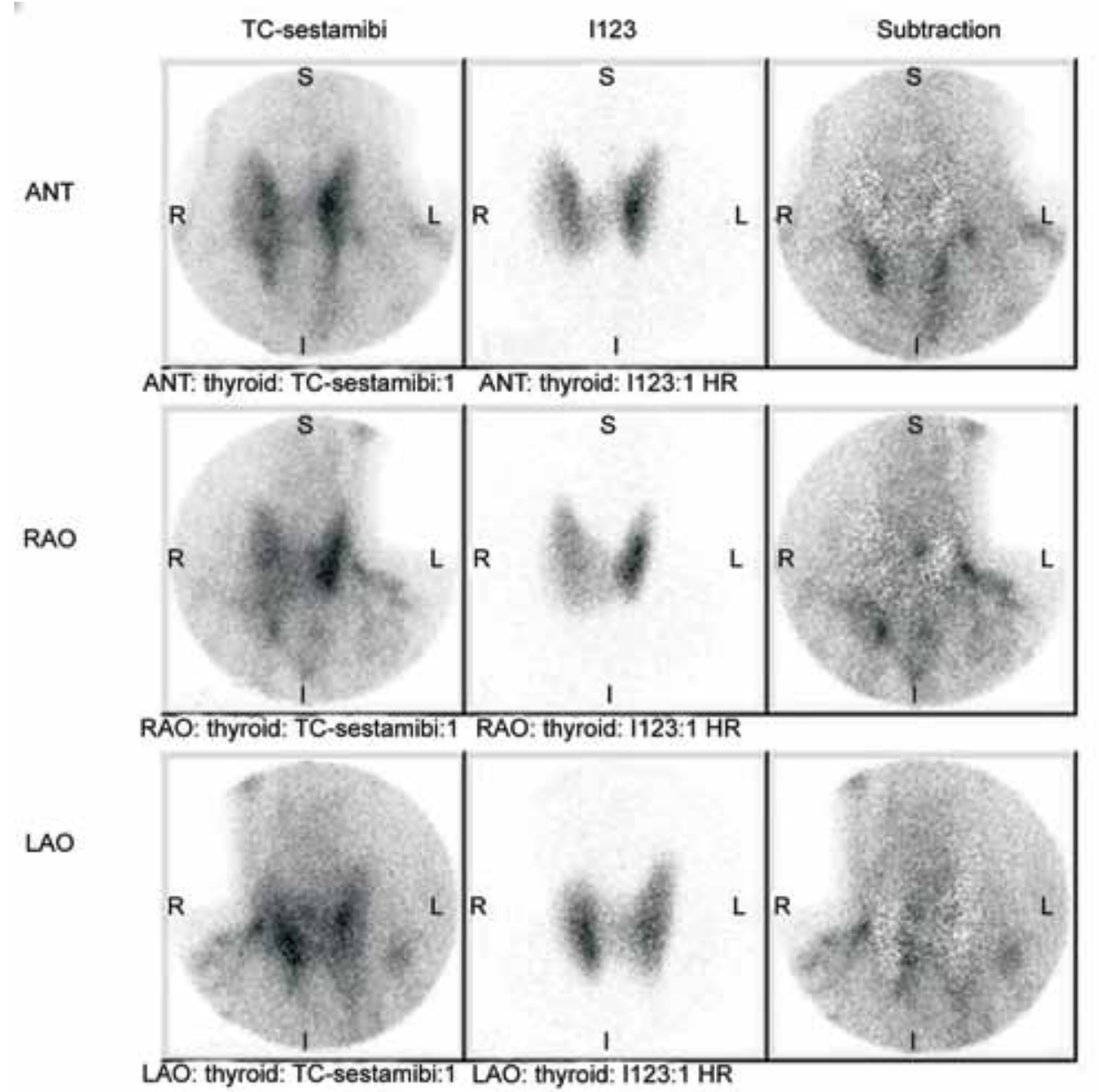

Fig. 2: Parathyroid sestamibi-single-photon emission computed tomography (SPECT)—parathyroid localization using a nuclear medicine parathyroid SPECT-CT shows hyperplasia inferiorly on anterior view 


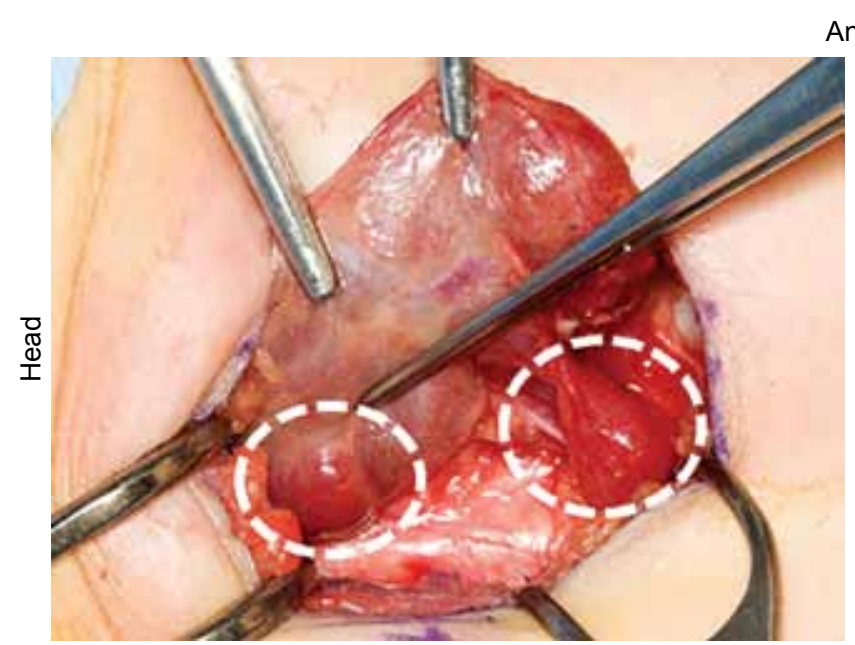

Right-sided exploration

Anterior

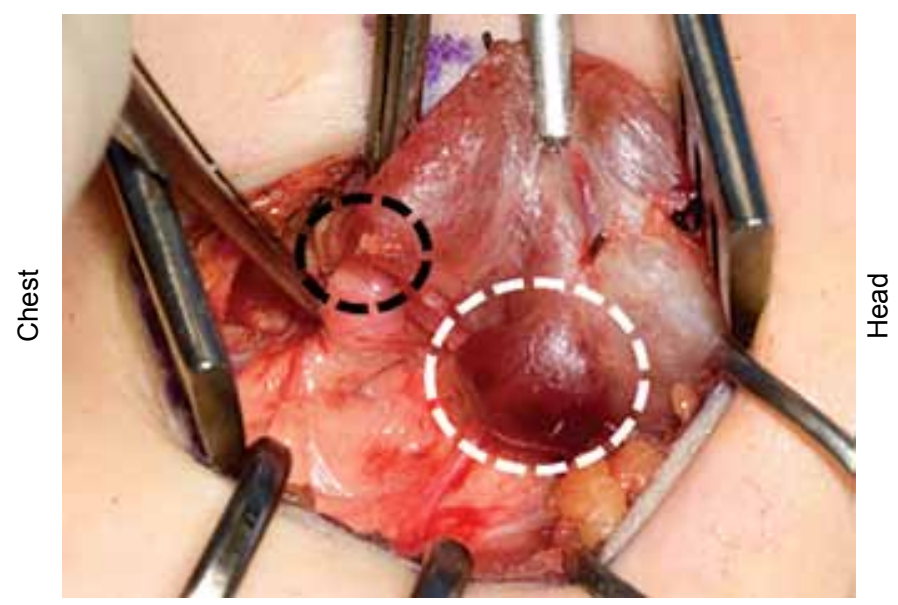

Left-sided exploration

Fig. 3: Subtotal parathyroidectomy—bilateral cervical exploration reveals three enlarged parathyroids (denoted by the white dashed circle). The left inferior parathyroid was of normal size (denoted by black dashed circle). In total, 3.5 glands were removed in this patient

this translates into decreased gastrointestinal tone. ${ }^{1,2,7-9}$ When this occurs at the level of the stomach, nausea and emesis can occur.

Regardless of the specific molecular etiology of nausea, parathyroidectomy for HPT has been proven to reduce subjective symptomatology and improve quality of life for patients. ${ }^{11}$ Resolution of general gastrointestinal symptoms, such as nausea have been reported to occur as early as 1 week after parathyroidectomy, although most patients demonstrate peak improvement at 6 weeks. ${ }^{12}$ Our patient experienced subjective improvement of nausea both when she was on cinacalcet and after parathyroidectomy. Her symptoms were associated with an elevated calcium level, however due to the use of a calcimimetic (which reduces both PTH and calcium); it is difficult to know whether the primary driver was PTH or calcium. However, as previously mentioned, hypercalcemia is the end-result of elevated PTH and calcium inhibits sympathetic enteric ganglia. Therefore, at the present time, calcium should be considered the primary culprit. Therapies for symptom reduction should target hypercalcemia. Further molecular studies are needed to identify specific gastrointestinal physiologic pathways affected by PTH.

\section{REFERENCES}

1. St Goar WT. Gastrointestinal symptoms as a clue to the diagnosis of primary hyperparathyroidism: a review of 45 cases. Ann Intern Med 1957; 46(1):102-118.
2. Abboud B, Daher R, Boujaoude J. Digestive manifestations of parathyroid disorders. World J Gastroenterol 2011;17(36): 4063-4066.

3. Chan AK, Duh QY, Katz MH, et al. Clinical manifestations of primary hyperparathyroidism before and after parathyroidectomy. A case-control study. Ann Surg 1995; 222(3):402-412.

4. Ming-Kai C, Ting Z. Primary hyperparathyroidism presenting as intractable nausea and vomiting. South Med J 2010; 103(11):1163-1166.

5. Pachydakis A, Koutroumanis P, Geyushi B, et al. Primary hyperparathyroidism in pregnancy presenting as intractable hyperemesis complicating psychogenic anorexia: a case report. J Reprod Med 2008;53(9):714-716.

6. Ellis C, Nicoloff DM. Hyperparathyroidism and peptic ulcer disease. Arch Surg 1968;96(1):114-118.

7. Gardner EC Jr, Hersh T. Primary hyperparathyroidism and the gastrointestinal tract. South Med J 1981;74(2):197-199.

8. Macdonald WC, Mc IH, Bogoch A. Gastrointestinal manifestations of hyperparathyroidism. Canadian Med Association J 1959;81(1):33-35.

9. Ebert EC. The parathyroids and the gut. J Clin Gastroenterol 2010;44(7):479-482.

10. Lindner G, Felber R, Schwarz C, et al. Hypercalcemia in the ED: prevalence, etiology and outcome. Am J Emerg Med 2013; 31(4):657-660.

11. Pasieka JL, Parsons L, Jones J. The long-term benefit of parathyroidectomy in primary hyperparathyroidism: a 10-year prospective surgical outcome study. Surg 2009;146(6):10061013.

12. Murray SE, Pathak PR, Pontes DS, et al. Timing of symptom improvement after parathyroidectomy for primary hyperparathyroidism. Surg 2013;154(6):1463-1469. 